literature investigating less common cardiovascular conditions, illustrating the need for future work if a more thorough picture of prevalence is to be established.

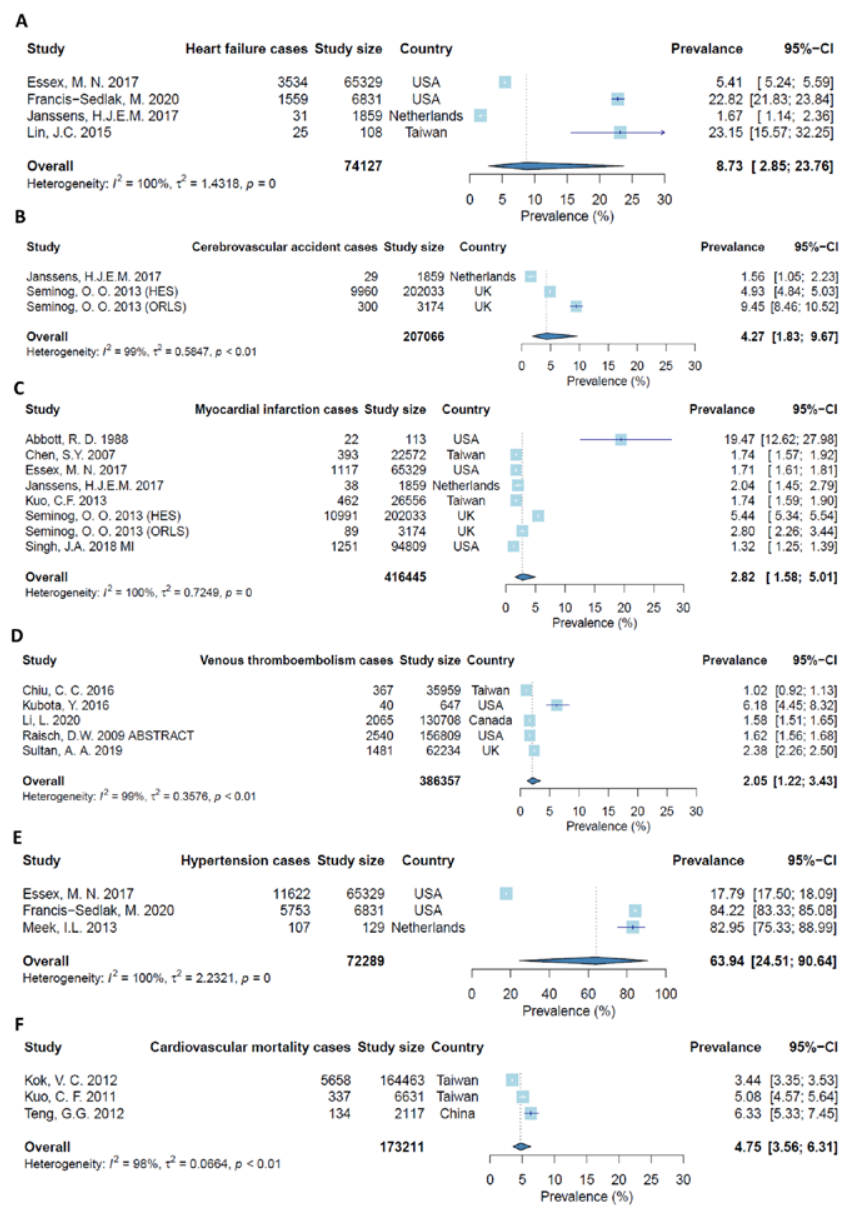

Figure 1. Forest plots of pooled prevalence of: (A) $8.73 \%$ for heart failure, (B) $4.27 \%$ for cerebrovascular accident, (C) $2.82 \%$ for myocardial infarction, (D) $2.05 \%$ for venous thromboembolism, (E) $63.94 \%$ for hypertension and (F) $4.75 \%$ for cardiovascular mortality.

Disclosure of Interests: None declared.

DOI: 10.1136/annrheumdis-2021-eular.1425

\section{POS1132 DIAGNOSTIC ACCURACY OF CONVENTIONAL RADIOGRAPHY OFTHE KNEE FOR CALCIUM PYROPHOSPHATE DEPOSITION DISEASE: AN ANCILLARY STUDY OF THE OMERACT ULTRASOUND - CPPD GROUP}

S. Sirotti ${ }^{1}$, F. Becce ${ }^{2}$, L. M. Sconfienza ${ }^{3}$, C. Pineda ${ }^{4}$, M. Gutierrez ${ }^{4}$, T. Serban ${ }^{5}$, D. Maccarter ${ }^{6}$, A. Adinolfi ${ }^{7}$, E. Naredo ${ }^{8}$, A. Scanu ${ }^{9}$, C. A. Scirè ${ }^{10}$, I. Möller ${ }^{11}$, P. Sarzi-Puttini ${ }^{1}$, A. Abhishek ${ }^{12}$, H. Choi ${ }^{13}$, N. Dalbeth ${ }^{14}$, S. Tedeschi ${ }^{15}$, M. A. D'agostino ${ }^{16}, \mathrm{H}$. Keen ${ }^{17}$, L. Terslev ${ }^{18}$, A. lagnocco ${ }^{19}$, G. Filippou on behalf of OMERACT Ultrasound working group, subgroup CPPD. ${ }^{1}$ Sacco Hospital, Rheumatology Department, Milano, Italy; ${ }^{2}$ Lausanne University Hospital, Department of Diagnostic and Interventional Radiology, Lausanne, Switzerland; ${ }^{3}$ University of Milan, Department of Biomedical Sciences for Health, Milano, Italy; ${ }^{4}$ National Rehabilitation Institute, Division of Musculoskeletal and Rheumatic Diseases, Ciudad de México, Mexico; ${ }^{5}$ Hospital La Colletta, Rheumatology Department, Arenzano, Italy; ${ }^{6}$ North Valley Hospital, Rheumatology Department, Whitefish, United States of America; ${ }^{7}$ Niguarda, Rheumatology Unit, Milan, Italy; ${ }^{8}$ Hospital Universitario Fundación Jiménez Díaz, Department of Rheumatology and Joint and Bone Research Unit, Madrid, Spain: ${ }^{9}$ University of Padua, Department of Medicine-DIMED, Rheumatology Unit, Padova, Italy: ${ }^{10}$ Società Italiana di Reumatologia, Epidemiology Research Unit, Milan, Italy; ${ }^{11}$ Instituto Poal de Reumatologia, Rheumatology, Barcelona, Spain; ${ }^{2}$ University of Nottingham, Academic Rheumatology, Nottingham, United
Kingdom; ${ }^{13}$ Massachusetts General Hospital Department of Medicine, Division of Rheumatology, Allergy, and Immunology, Boston, United States of America;

${ }^{14}$ The University of Auckland, Bone and Joint Research Group, Department of Medicine, Auckland, New Zealand; ${ }^{15}$ Brigham And Women's Hospital, Division of Rheumatology, Inflammation and Immunity, Boston, United States of America; ${ }^{16}$ Agostino Gemelli University Policlinic, Rheumatology Department, Roma, Italy: ${ }^{17}$ The University of Western Australia, School of Medicine, Murdoch, Perth, Australia: ${ }^{18}$ Rigshospitalet, Center for Rheumatology and Spine Diseases, København, Denmark; ${ }^{19}$ University of Turin, Academic Rheumatology Centre, Department of Clinical and Biological Sciences, Torino, Italy

Background: Conventional Radiography (CR) has been widely used in the assessment of knee chondrocalcinosis (CC) and is still considered one of the most important diagnostic methods for the diagnosis. However, there are very few studies that examine the diagnostic accuracy of CR compared to histology of the knee tissues.

Objectives: To assess the diagnostic accuracy of $\mathrm{CR}$ of the knee in Calcium Pyrophosphate Deposition Disease (CPPD) by using the recently created definitions for CPPD in CR of the ACR/EULAR taskforce for the new classification criteria for CPPD.

Methods: This is an ancillary study of the Criterion Validity of Ultrasound in CPPD study [1]. Consecutive patients with osteoarthritis $(\mathrm{OA})$ awaiting total knee replacement were enrolled in 4 centres from Romania, Italy, USA and Mexico. All patients underwent $\mathrm{CR}$ of the knees taken maximum 6 months before surgery, in posterior-anterior weight baring and lateral projections. DICOM files of the radiographs were anonymised and read independently by two musculoskeletal radiologists with experience in microcrystalline arthropathies. For each patient, a dichotomic score was used (absence/presence of $\mathrm{CC}$ ) at the level of the menisci and tibiofemoral hyaline cartilage by each reader. The definitions of the ACR/EULAR taskforce for identification of CPPD in CR were used in this study [paper in preparation]. According to these definitions CPPD in CR appears as "linear or punctate opacities in the region of fibro- or hyaline articular cartilage/synovial membrane or joint capsule/ within tendons or entheses that are distinct from denser, nummular radio-opaque deposits due to basic calcium phosphate deposition." In case of disagreement a consensus decision was taken by both radiologists after discussion of the case. Menisci and the hyaline cartilage were analysed using compensated polarized ligh microscopy as described previously [1], patients were considered positive for CPPD if at least one of their tissue specimens revealed the presence of calcium pyrophosphate crystals. All examiners were blind to each other's findings.

Results: We enrolled 33 patients with OA (61\% female, mean age $69 y$ y). The accuracy values of $C R$ in the various sites of the knee are indicated in Table 1. CR demonstrated to be a specific exam for identification of CPPD at the knee, but sensitivity remains low in all sites and in the overall evaluation. Identification of CPPD appears challenging and this could be due to the advanced grade of $\mathrm{OA}$ in our cohort of patients. Advanced degeneration, dislocation of the menisc and thinning of the hyaline cartilage in these patients is frequent and the eventual presence of calcific deposits in one of these structures could overlap with other anatomical structures making the exact localisation difficult. According to the results of the predictive values, the presence of typical deposition on $\mathrm{CR}$ allows a definite confirmation of the diagnosis, but a negative radiography does not exclude CPPD as testified by the low negative predictive value.

Table 1. Sensitivity, specificity, PPV, NPV, accuracy and AUC of CR for identification of CPPD by using the new ACR/EULAR taskforce definitions.

\begin{tabular}{lcccc}
\hline & Medial meniscus & Lateral meniscus & Hyaline cartilage & Overall \\
\hline Sensitivity & $22 \%$ & $33 \%$ & $31 \%$ & $42 \%$ \\
Specificity & $100 \%$ & $100 \%$ & $85 \%$ & $90 \%$ \\
Positive predictive value & $100 \%$ & $100 \%$ & $67 \%$ & $80 \%$ \\
Negative predictive value & $56 \%$ & $60 \%$ & $55 \%$ & $61 \%$ \\
Accuracy & $61 \%$ & $68 \%$ & $58 \%$ & $66 \%$ \\
AUC & 0.6 & 0.7 & 0.6 & 0.7
\end{tabular}

Conclusion: CR has been extensively used for the diagnosis of OA and CPPD and has been tested previously for diagnostic accuracy. The results of our study confirm that the presence of typical CPPD calcifications, as defined by the ACR EULAR task force, are highly specific but have low sensitivity for disease identification when using CR. Absence of CPPD on CR does not exclude the diagnosis. REFERENCES:

[1] Filippou G, et al. Criterion validity of ultrasound in the identification of calcium pyrophosphate crystal deposits at the knee: an OMERACT ultrasound study. Ann Rheum Dis 2020. doi:10.1136/annrheumdis-2020-217998

Disclosure of Interests: None declared.

DOI: 10.1136/annrheumdis-2021-eular.1437 